\title{
PLC Programming Case Study for Hydraulic Positioning Systems Implementations
}

\author{
M. Papoutsidakis \\ Dept. of Automation \\ Engineering \\ PUAS, Athens, Greec
}

\author{
A. Xatzopoulos \\ Dept. of Automation \\ Engineering \\ PUAS, Athens, Greece
}

\author{
G. P. Smyraiou \\ Dept. of Automation \\ Engineering \\ PUAS, Athens, Greece
}

\author{
D. Tseles \\ Dept. of Automation \\ Engineering \\ PUAS, Athens, Greece
}

\begin{abstract}
With the rapid and vertiginous development of technology of hydraulic automation systems is observed in our current era, an ongoing effort to introduce engineering technology, automated solutions in everyday life with an emphasis on the field of hydraulic automation systems. The systems sector develops increasingly their fields of application, whether simple or hydraulic circuits for modern hydraulic automation circuits. In the systems with automated processes, the systems sector with the help of have only recently programmable logic controller has begun to make their appearance. In this dissertation, the structure of a hydraulic circuit which has the purpose control and observation of the hydraulic system with the aid of a P.L.C. Moreover, it describes the properties of the hydraulic system, its structural components and the detailed functional description of Programmable Logic Controller. Finally, the work ends with the recording of the findings of the use of this integrated hydraulic circuit.
\end{abstract}

\section{Keywords}

Programming language, hydraulic systems, hydraulic cylinders, hydraulic valves, hydraulic pumps, hydraulic actuators, programmable logic controller

\section{INTRODUCTION}

The requirements of modern times and particularly the last five years have imposed a rapidly developing technology in the hydraulic automation area. As a result, a new technology sector appeared: hydraulic. This remarkable growth is mainly due to the contribution of the construction of hydraulic components and P.L.C, general.

By hydraulic system we mean that a device used to transmit motion and power transmission from the motor to the driven machine. The analysis, design and modeling hydraulic automation systems, based on principles and laws, is widely applicable in fields such as the automotive industry and in robotics [1].

Typical properties that consist a hydraulic system are the following:

- Fluid density: The density of a fluid is the mass of the fluid per unit volume in a particular location of the fluid. If the fluid is incompressible, the density remains the same at all points of the fluid force: $\mathrm{p}=\mathrm{m} / \mathrm{v}[1]$.

- Specific weight: The specific weight of a fluid is the fluid weight per unit volume, and it is as follows: $\mathrm{C}=\mathrm{B} / \mathrm{v}=\mathrm{mg} / \mathrm{v}=\mathrm{p} * \mathrm{~g}$.

- Special pressure: The pressure is defined as the ratio of the force exerted by the fluid on a surface to the

$$
\begin{aligned}
& \text { area of that surface. } \\
& \text { So the pressure is given by the following form: } \\
& \mathrm{P}=\mathrm{F} / \mathrm{A} \text {. }
\end{aligned}
$$

- Hydraulic capacity: The hydraulic capacity of a hydraulic size is associated with energy storage. It is defined as the ratio of the change in the volume of fluid in a hydraulic device to the pressure variation. For the calculation of the hydraulic capacity it is necessary to produce the ratio of pressure to flow in the particular application.

- Hydraulic inertia: The hydraulic inertia is due to inertia presenting a fluid to changes in speed. It is defined as the ratio of the pressure difference in the change of flow rate.

- Hydraulic resistance: The hydraulic resistance is the resistance encountered by the fluid during the movement of the pipelines. It expresses the ratio of the pressure difference to the volume flow. The hydraulic resistance depends on the shape of the fluid flow.

A hydraulic automation system brings advantages and disadvantages [2].

The advantages are:

- Variable speed:

Common hydraulic motors have the possibility to change the speed.

- Protection against overloads and control of loads:

The hydraulic valve in a hydraulic automation system protects the system from an uncontrolled increase of the load.

- Small and limited volume:

Hydraulic components and the whole hydraulic system provides high performance by combining small size and light weight.

- Wide range of control like, great possibilities to control a hydraulic circuit automation. Monitoring can be automated with the help of a P.L.C, manually using a start button, electronically controlled by means of a sensor or a combination of all.

The disadvantages are:

- Small tolerances as the structures with high accuracy result in the hydraulic components creating cost prohibitive.

- Heat dissipation, as the heat released due to internal leakage is a limit for every machine 
- Plumbing problems like that there are no laws for the design of hydraulic circuits, as for example, it can be said electrical circuits. That is, there is nothing like Ohms Law in the behavior of hydraulic fluids.

\section{BASIC COMPONENTS}

As hydraulic automation systems we can define transfer systems, which consist of hydraulic lines, hydraulic pumps, hydraulic motors and hydraulic pistons used to generate power or fluid torque. By fluid flow we refer to the movement of the fluid particles, because of flowing, but which piece includes the concepts of the force that causes the movement, speed and acceleration of the fluid. In hydraulic systems the power transmission is performed using mainly incompressible fluid such as oil [2]. An integrated hydraulic automation system consists of the following components:

- Hydraulic cylinders

- Hydraulic valves

- Hydraulic pumps

- Hydraulic actuators

Hydraulic cylinders:

Hydraulic cylinders are classified as single and double acting cylinders, which are the most used. In addition, there are a large number of cylinder variants, each one designed for a specific use. For example it is telescopic cylinders through which path rod several times its original length is achieved [3], [4].

The hydraulic valves are key components of hydraulic automation systems for regulating the position, acceleration of a hydraulic piston. These valves are divided into two major categories: the hydraulic valves as how to set and hydraulic valves as mode. Valves as how to set regulate fluid flow.

The hydraulic valves are divided according to the flowregulating manner in the following categories:

- Piston type valves:

Here the hydraulic valves to regulate flow is achieved by movement of a piston which permits the passage or the stoppage of the hydraulic fluid from the valve ports.

- Nozzle flap type valves:

The hydraulic valves are based on the principle of variable leakage achieved by moving a blade.

While the valves as our mode show how the valve operates and how it conveys the flow to the other structural components of the hydraulic system.

The categories on the principle of operation is as follows:

- Directional valves or valves drivers:

Hydraulic directional valve in a hydraulic circuit, which with the appropriate hydraulic actuation determine the direction of flow to a piston.

The hydraulic valves are limiting the hydraulic pressure in hydraulic automation systems, which provide an escape passage of the hydraulic fluid to the reservoir. The hydraulic valves protect hydraulics from the great pressure that can be brought to them from the pump. Flow Control Valves: The flow control valves are used for regulating the flow in hydraulic automation. The hydraulic valves are used in hydraulic systems which have constant pressure and do not have a different flow. A last structural hydraulic component contained in the system is the hydraulic actuator, or otherwise sensing hydraulics. Hydraulic actuators are devices that detect the movement, velocity, displacement and the power of a hydraulic piston. They receive data and information from P.L.C or hydraulic valves and give relevant signal through the acceleration, the displacement, the position or the speed of the hydraulic piston [5].

The essential characteristics of a hydraulic sensor are: - Operating Range: The limits to which the hydraulic automation system works reliably.

- Accuracy: The proximity of the output value to the input value.

- Error: The difference between the measured value and actual value.

- Delay: The delay of the change of the output to the input.

- Repeatability: Producing the same effect at different times with the same input.

- Operating time: The estimated operation time as part of the hydraulic circuit specifications.

Hydraulic actuators are divided into four categories and are as follows [5], [6]:

- Displacement and motion sensors:

These sensors determine the displacement and movement of a hydraulic piston, ie to what extent a hydraulic piston is moved or rotated.

Displacement sensors and drive a hydraulic piston is:

$\neg$ Linear potentiometer

$\neg$ Goniometer variable reluctance

$\neg$ Linear reed switch

- Power Sensors:

The use of these sensors is capable of measuring the power of a hydraulic piston.

The one piston force sensors are divided into two types, which are:

$\neg$ The load cells

$\neg$ The spring yoke

- Detection sensors:

The sensors detect the piston ie whether the hydraulic piston is located inside or outside the range.

The one piston sensors are divided into three categories and are as follows:

$\neg$ inductive sensors

$\neg$ Capacitive Sensors

$\neg$ Magnetic sensors

- Optical indication sensors:

The use of these sensors is to show us various information necessary for the hydraulic piston.

The visual indication sensors or optoaisthitires a hydraulic piston is:

$\neg$ The manometer

$\neg$ Sensor reflected beam

All the above briefly described components connected can be illustrated in the below Figure 1: 


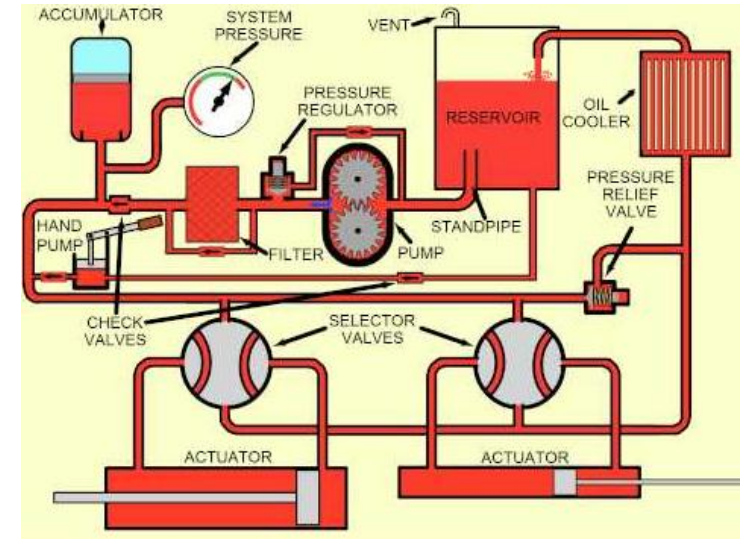

Fig 1: A Typical Hydraulic System

For this given research project the hydraylic system which was used for the experimentation can be provided in the below schematic diagram.

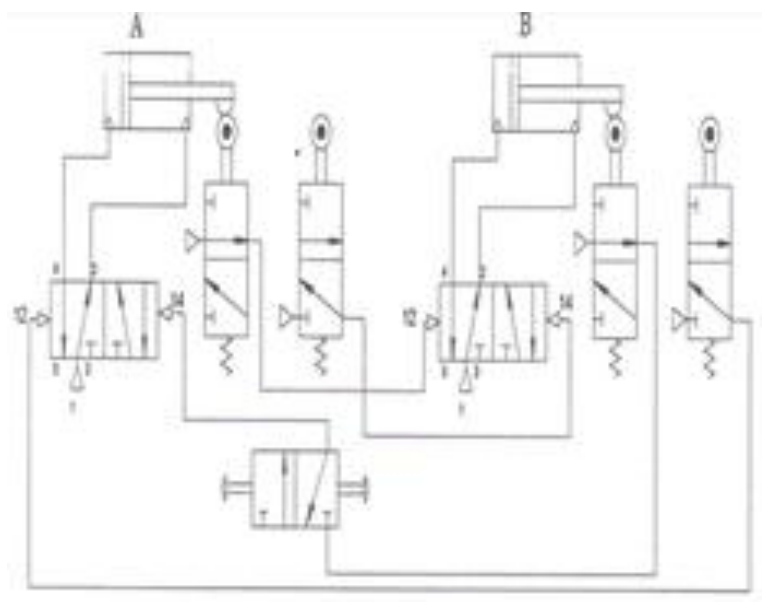

Fig 2: The Hydraulic System of the Task

\section{PLC STRUCTURE AND HYRDAYLIC RIG IMPLEMENTATION}

The most significant part of the paper is the connectivity and the programming of a PLC in order to operate the system automatically. The PLC model which was selected for the task is LOGO P.L.C. Generally P.L.C is a digital microprocessor that is tailored to implement any kind of automation from the start of a plunger to the full operation of the hydraulic circuit automation.

A P.L.C is able to perform the calculation and communication tasks. As the device is very flexible, strong, can be assembled at low cost and has some advantages [7]:

- The construction time of automation.

- The PLC is flexible in modifying the operation of the hydraulic automation, where a change in automation is a few minutes, changing only the program.

- Minimize maintenance costs, since there is no question of failure because the PLC rarely breaks down.

- The structure of a programmable logic controller is uniform and consists of the following components: $\neg$ Units Mounting Frame

$\neg$ Automation Units

$\neg$ Power Unit

$\neg$ Central processing unit

$\neg$ Real time clock

$\neg$ Developer

The key piece to a hydraulic circuit with PLC automation is not the structural part, that is, the application simulation but the software, ie the program that implements the desired hydraulic automation system. Using commands to create a program, determines the correct or not operation of a device. The way that these orders will be transferred to specific PLC, is called a programming language [7]. There are generally three categories of programming languages for P.L.C, which are:

-LAD language or languages electrical graphics -STL language or language of logic commands -FDB language or language graphics logic

The placement of the LOG PLC inside the experimental circuitry is provides in the next figure in an attempt to highlight the ports connectivity of the PLC and the way the PLC actually generates an interface between the system and the user.

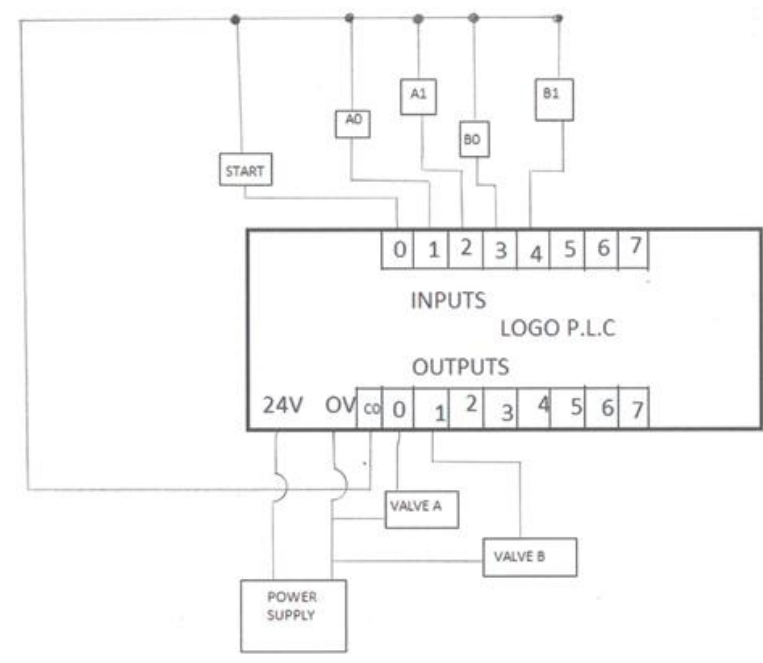

Fig 3: LOGO PLC connections

The experimentation rig of the project relates to an integrated hydraulic automation system. The hydraulic circuit of automation is associated with two hydraulic pistons that make some movements and operation of the hydraulic circuit begins with a start switch, which is based on the operation string of Figure 2:

$\mathrm{A}+, \mathrm{B}+, \mathrm{A}-, \mathrm{B}-$

The first programming language designed for the above operation scenario was LADDER and the control algorithm that follows classical electrical logic is illustrated in Figures 4 and 5 . 


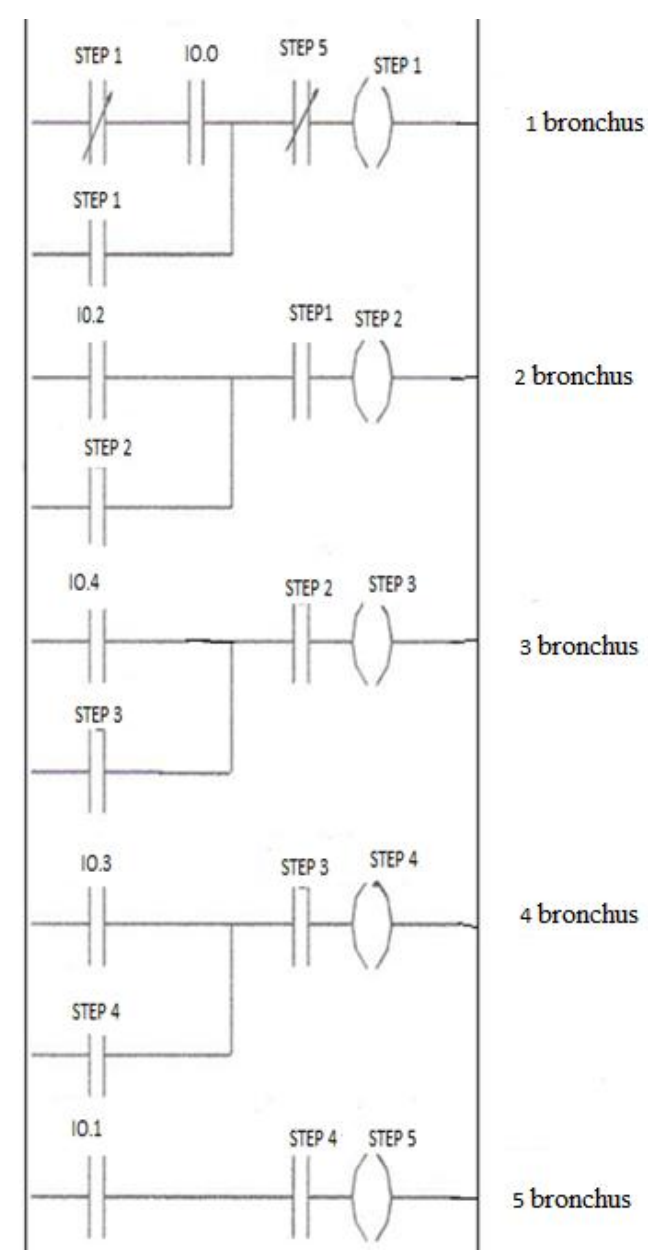

Fig 4: LADDER language programing (part1)

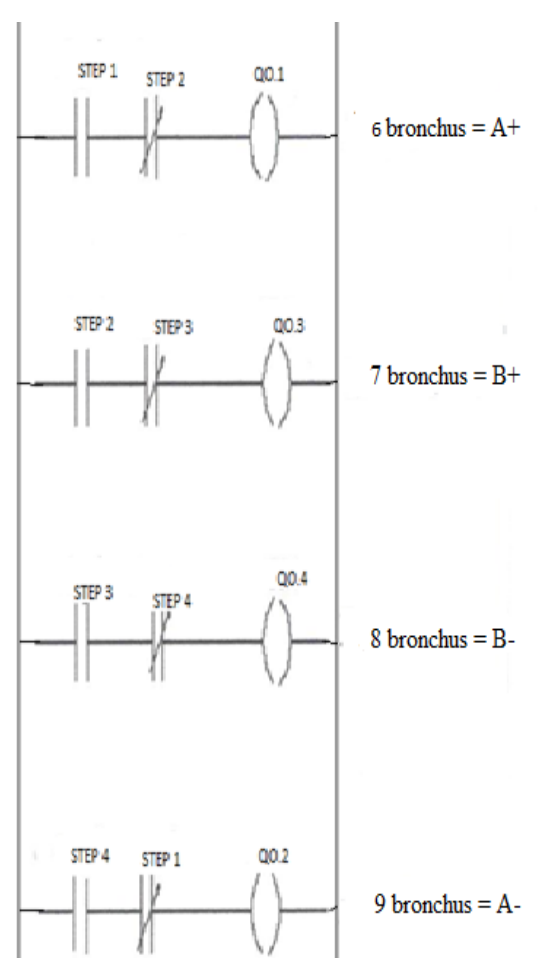

Fig 5: LADDER language programing (part2)
On the other hand, FBD language and its language graphics logic can be shown right below:
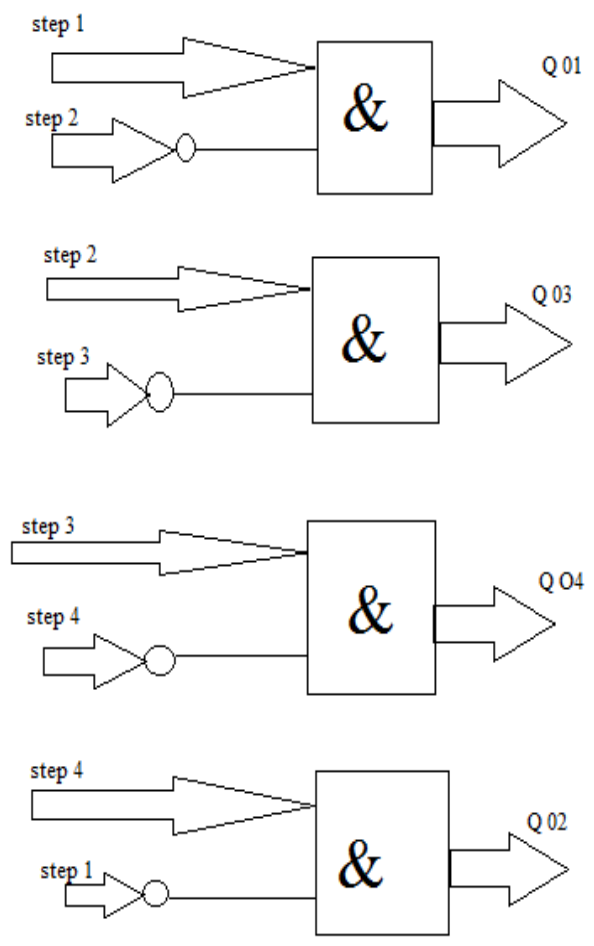

Fig 6: FBD language programing

STL language or language of logic commands

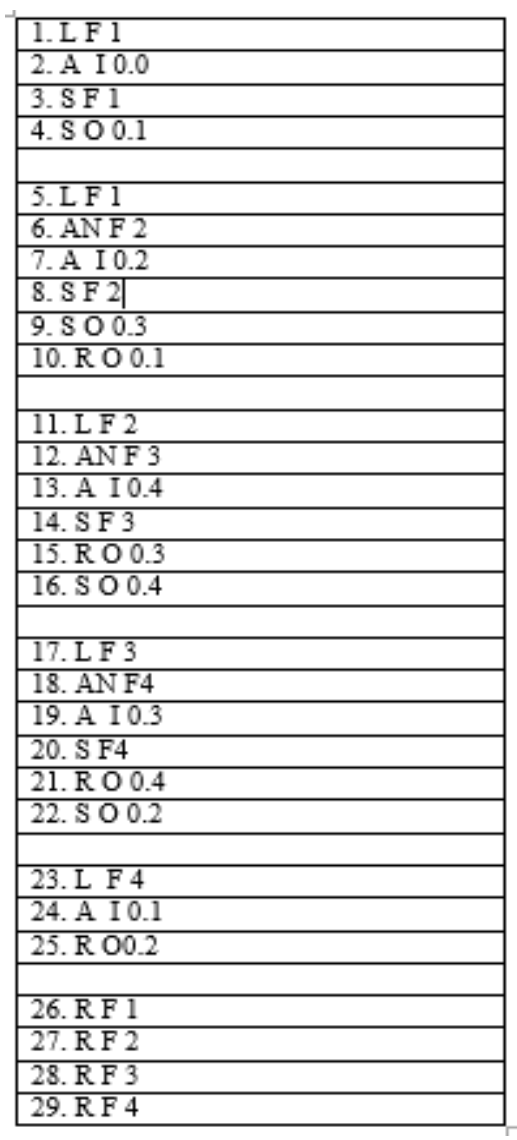

Fig 7: FDB language programing 


\subsection{Industrial Wireless Sensor Network (IWSNs) and Security}

Wireless sensor solutions are now being used in countless situations where it is necessary to monitor remote, difficult, and costly to reach locations, or moving applications. When choosing the best wireless approach, there are many technology tradeoffs and vendors to consider [12-13]. See Tables 1 below, which offer means to compare network selection criteria according to application needs.

\section{CONCLUSIONS AND FUTURE IMPROVEMENTS}

This paper tries to show the usefulness, design, modeling and the importance of hydraulic automation systems in the industrial workplace. The experimentation highlights to an engineer how to implement PLC technology with 3 different programming languages and also illustrated its reliability. In details the first step was about the theoretical background of building hydraulic components which contribute to an integrated automation system. In addition a second step here was comprehensive and detailed analysis of Programmable Logic Controller and its implementation.

Of course there is room for future improvements, which can indicatively be, voice control a hydraulic circuit automation and automatic adjustment of structural mechanical parts on a hydraulic circuit.

\section{ACKNOWLEDGMENTS}

All authors would like to express their gratitude to the PostGraduate Program of Studies "Automation of Productions and Services" of PUAS, for the financial support to undertake this research project

\section{REFERENCES}

[1] Hydraulic automatic control systems, N. Pantazis, Publisher ION

[2] Electricity, Fluid Power, and Mechanical Systems for Industrial Maintenance, Thomas E. Kissell, 1st Edition

[3] Hydraulic Control Systems, Herbert E. Merritt

[4] Hydraulic and Pneumatic Systems, Theodore N. Costopoulos, Simeon Publications, Athens 2009

[5] Automatic Control and Automation, N. Pantazis, Publisher Stamouli

[6] Modern Industrial and Electrical Motor Controls, Thomas E. Kissel

[7] Automation programmable controllers, Stavros Roumpo, Publisher Simeon 